\title{
Dichloroacetate is an effective treatment for sarcoma models in vitro and in vivo
}

\author{
Melissa Rooke1, Lucy A Coupland ${ }^{1}$, Thy Truong², Anneke C Blackburn ${ }^{1 *}$ \\ From Metabolism, Diet and Disease 2014: Cancer and metabolism \\ Washington DC, USA. 28-30 May 2014
}

\section{Background}

Sarcomas are cancers that arise from tissues of mesenchymal origin and there has been limited improvement in treatments over the last 30 years. The Warburg effect is a widespread metabolic phenotype of cancer, where glycolysis is favoured despite the presence of oxygen. Dichloroacetate (DCA) is a pyruvate dehydrogenase kinase (PDK) inhibitor in clinical use that can reverse the Warburg effect, inhibiting growth and enhancing apoptosis in a range of cancers. We have investigated its effectiveness against sarcoma cells in vitro and in vivo.

\section{Materials and methods}

Three cell lines (mouse fibrosarcoma S180, mouse osteosarcoma K7M2 and human fibrosarcoma HT1080-luc2) were examined for cell viability after DCA treatment in vitro (neutral red uptake assay), alone and in combination with doxorubicin. In vivo, K7M2 cells were injected s.c. into $\operatorname{Rag} 1^{-1-}$ mice (2 sites per mouse, 7-9 mice per group) and established tumours were treated with DCA in the drinking water $(0,0.5,1.0$ and $1.5 \mathrm{~g} / \mathrm{L}$, delivering 0,70 , 125 and $165 \mathrm{mg} / \mathrm{kg} /$ day, respectively). Tumour growth was monitored with callipers. Plasma was collected on $\mathrm{d} 1$ and $\mathrm{d} 15$ for measurement of DCA levels (LC-MS).

\section{Results}

DCA significantly reduced the total viable cell number after $48 \mathrm{~h}$ of treatment in the mouse sarcoma lines $(\sim 15 \%$ at $0.5 \mathrm{mM} \mathrm{DCA}$, and $30-40 \%$ at $5 \mathrm{mM} \mathrm{DCA})$, however HT1080-luc2 cells showed only a $10 \%$ reduction in cell number with $5 \mathrm{mM}$ DCA. There was no morphological indication of apoptosis, suggesting DCA was decreasing proliferation. Chronic treatment of the mouse cells $(5 \mathrm{mM}$ DCA for 2 weeks) resulted in significantly slower growth rates as measured over $48 \mathrm{~h}$ (7 and 13\% total cell number compared to untreated S180 and K7M2 cells, respectively). DCA did not synergise with doxorubicin but was additive at lower concentrations of doxorubicin. In vivo, 1.0 and $1.5 \mathrm{~g} / \mathrm{L}$ DCA significantly reduced tumour growth $(33.0$ and $33.1 \%$ reduction in tumour size on $\mathrm{d} 13, \mathrm{p}=0.001$ and 0.01 respectively). Plasma DCA was undetectable on $\mathrm{d} 1$ of treatment, but by $\mathrm{d} 15,1.0$ and $1.5 \mathrm{~g} / \mathrm{L}$ DCA delivered 2-44 uM. Tumour DCA concentrations were also measured and found to be in the range of 25-470 uM, much lower than those typically used in in vitro studies.

\section{Conclusions}

DCA was effective against an in vivo sarcoma model, with tumour DCA concentrations in the micromolar range. These concentrations are achievable clinically, thus DCA warrants further investigation for sarcoma treatment.

\section{Acknowledgements \\ Supported by the Sarah-Grace Sarcoma Foundation and Cancer Australia. \\ Authors' details \\ 'John Curtin School of Medical Research, Australian National University, Canberra, ACT, Australia. ${ }^{2}$ Mass Spectrometry Facility, Australian National University, Canberra, ACT, Australia.}

Published: 28 May 2014

doi:10.1186/2049-3002-2-S1-P9

Cite this article as: Rooke et al:: Dichloroacetate is an effective

treatment for sarcoma models in vitro and in vivo. Cancer \& Metabolism 2014 2(Suppl 1):P9. 